\title{
Guest editorial: role of monoclonal antibodies for the prevention and treatment of graft-versus-host disease
}

\author{
Yoshinobu Kanda
}

Received: 17 April 2011/Revised: 21 April 2011/Accepted: 21 April 2011/Published online: 11 May 2011

(C) The Japanese Society of Hematology 2011

\begin{abstract}
Allogeneic hematopoietic stem cell transplantation (HSCT) has been established as an effective treatment for hematological disorders. However, graft-versus-host disease (GVHD) remains a major problem following allogeneic HSCT. The development of GVHD is strongly associated with an increased transplant-related mortality and impaired quality of life. The development of GVHD, however, may also decrease the incidence of relapse of hematological malignancies through the graft-versus-leukemia/lymphoma (GVL) effect. Nevertheless, the increased transplantedrelated mortality due to GVHD overweighs the benefit of the GVL effect in most patients, as shown in one Japanese study [1], and strategies for the prevention or treatment of GVHD remain a major issue in an effort to improve HSCT outcome. The ideal level of immunosuppression for the control of GVHD can be difficult to determine, as excessive immunosuppression may increase the incidence of disease relapse and infectious complications. In addition, each immunosuppressant has its own toxicities such as renal toxicity associated with the use of calcineurin inhibitors.
\end{abstract}

The combination of cyclosporine (CSA) and methotrexate (MTX) has been established as a standard regimen for the prophylaxis against acute GVHD based on a randomized controlled trial performed by the Seattle group [2]. Subsequently, three randomized trials showed that tacrolimus (TAC) is more effective than CSA in preventing acute GVHD, although no survival benefit was observed, due to increased toxicity in the TAC group [3]. There was a

\section{Y. Kanda $(\square)$}

Division of Hematology, Saitama Medical Center,

Jichi Medical University, 1-847 Amanuma-cho,

Omiya-ku, Saitama 330-8503, Japan

e-mail: ycanda-tky@umin.ac.jp significant concern regarding the target concentrations of CSA and TAC in these studies, however, and re-evaluation will be necessary [4]. Recently, mycophenolate mofetil (MMF) has been investigated as a partner of calcineurin inhibitors in the place of MTX. The results of small-scale randomized trials showed less mucositis in the MMF group than in the MTX group, but inconsistent results were observed among studies with regard to its efficacy in preventing acute GVHD.

Methylprednisolone (mPSL) or prednisolone (PSL) at $1-2 \mathrm{mg} / \mathrm{kg} /$ day is the standard initial treatment for acute GVHD, producing a response in $50-80 \%$ of patients [5]. The prognosis of patients with steroid-refractory acute GVHD, however, is extremely poor. Anti-thymocyte globulin (ATG) is one of the most widely used second-line agents for steroid-refractory acute GVHD [6], but ATG combined with high-dose mPSL was not found to be more efficacious than high-dose mPSL alone in one randomized controlled trial [7]. The first-line treatment for chronic GVHD is also based on $1 \mathrm{mg} / \mathrm{kg} /$ day of PSL [8]. Several uncontrolled trials produced encouraging results with the use of MMF as second-line treatment for steroid-refractory chronic GVHD, and in fact MMF is the most frequently used agents for steroid-refractory chronic GVHD, although its efficacy has not been tested in randomized controlled trials [6].

Recently, our understanding of the pathogenesis of GVHD has dramatically improved mainly through studies in mouse models [9], which have led to novel approaches to the management of GVHD. Numerous molecules identified as involved in the pathogenesis of GVHD are considered to be candidate targets for use in the prevention or treatment of GVHD. In the Progress in Hematology series in this issue of the International Journal of Hematology, four experts in the area of GVHD research review roles for 
monoclonal antibodies directed against such molecules in the prevention and treatment of GVHD. John E. Levine opens with a review on the implications of TNF- $\alpha$, a proinflammatory cytokine, in the pathogenesis of GVHD and introduces strategies for controlling GVHD using TNF- $\alpha$ as a therapeutic target. Next, Corey Cutler shows the impact of the use of rituximab on the incidence of acute GVHD, as well as the effect of second-line treatment by rituximab for steroid-refractory chronic GVHD. These clinical studies were based on a recent understanding of the important role of B lymphocytes in the pathogenesis of GVHD. David Rizzieri and colleagues provide a comprehensive review of the use of alemtuzumab, a monoclonal antibody against the CD52 antigen, mainly for the prevention of GVHD, including a recent investigation into haploidentical HLAmismatched HSCT without ex vivo $\mathrm{T}$ cell depletion. Finally, JK Davies describes an interesting strategy for enabling haploidentical HLA-mismatched HSCT by inducing the alloanergization of donor $\mathrm{T}$ cells via allostimulation and blockade of costimulatory signals. The study design of an ongoing clinical trial that based on the basic studies and a successful previous clinical study is explained in detail.

Although none of these treatments are indicated for the prevention or treatment for GVHD in Japan, I believe that these reviews will be of interest to readers of the International Journal of Hematology and provide insights into future directions in the management of GVHD following allogeneic HSCT.

\section{References}

1. Kanda $\mathrm{Y}$, Izutsu $\mathrm{K}$, Hirai $\mathrm{H}$, et al. Effect of graft-versus-host disease on the outcome of bone marrow transplantation from an HLA-identical sibling donor using GVHD prophylaxis with cyclosporin A and methotrexate. Leukemia. 2004;18:1013-9.

2. Storb R, Deeg HJ, Whitehead J, et al. Methotrexate and cyclosporine compared with cyclosporine alone for prophylaxis of acute graft versus host disease after marrow transplantation for leukemia. N Engl J Med. 1986;314:729-35.

3. Ram R, Gafter-Gvili A, Yeshurun M, Paul M, Raanani P, Shpilberg O. Prophylaxis regimens for GVHD: systematic review and meta-analysis. Bone Marrow Transplant. 2009;43:643-53.

4. Oshima K, Sato M, Terasako K, et al. Target blood concentrations of CYA and tacrolimus in randomized controlled trials for the prevention of acute GVHD after hematopoietic SCT. Bone Marrow Transplant. 2010;45:781-2.

5. Bacigalupo A. Management of acute graft-versus-host disease. $\mathrm{Br}$ J Haematol. 2007;137:87-98.

6. Lee SJ, Joffe S, Artz AS, et al. Individual physician practice variation in hematopoietic cell transplantation. J Clin Oncol. 2008;26:2162-70.

7. Van Lint MT, Milone G, Leotta S, et al. Treatment of acute graftversus-host disease with prednisolone: significant survival advantage for day +5 responders and no advantage for nonresponders receiving anti-thymocyte globulin. Blood. 2006;107:4177-81.

8. Wolff D, Gerbitz A, Ayuk F, et al. Consensus conference on clinical practice in chronic graft-versus-host disease (GVHD): first-line and topical treatment of chronic GVHD. Biol Blood Marrow Transplant. 2010;16:1611-28.

9. Ferrara JL, Cooke KR, Teshima T. The pathophysiology of acute graft-versus-host disease. Int J Hematol. 2003;78:181-7. 